\title{
Open Loop Optimal Control of Base Station Activation for Green Networks
}

\author{
Sreenath Ramanath ${ }^{1}$, Veeraruna Kavitha ${ }^{1,2}$ and Eitan Altman ${ }^{1}$ \\ ${ }^{1}$ INRIA, Sophia-Antipolis, France, ${ }^{2}$ Universite d'Avignon, Avignon, France
}

\begin{abstract}
In recent years there has been an increasing awareness that the deployment as well as utilization of new information technology may have some negative ecological impact. This includes awareness to energy consumption which could have negative consequences on the environment. In recent years, it was suggested to increase energy saving by deactivating base stations during periods in which the traffic is expected to be low. In this paper we study the optimal deactivation policies, using recent tools from Multimodularity (which is the analog concept of convexity in optimization over integers). We consider two scenarios: In the first case, a central control derives the optimal open loop policies so as to maximize the expected throughput of the system given that at least a certain percentage of Base stations are deactivated (switched OFF). In the second case, we derive optimal open loop polices, which each base station can employ in a decentralized manner to minimize the average buffer occupancy cost when the fraction of time for which the BS station is deactivated (idle mode) is lower bounded. In both the cases, we show that the cost structure is Multimodular and characterize the structure of optimal policies.
\end{abstract}

\section{INTRODUCTION}

In recent years, there is a paradigm shift towards greener and denser networks [1], [2]. While green networks aim at reducing overall power and energy consumption in networks, denser networks increase the capacity and coverage of networks. Typically, dense networks, popularly referred to as small cell networks comprise Femto and pico cells serving indoor, hot-spot and urban mobility regions. The base stations used in such cells are compact and small portable devices, which can be easily installed on existing infrastructure and are often battery operated. They recharge periodically and it would be beneficial from a greener perspective if this can be as less frequent as possible. Thus these base stations need to judiciously use the available battery power. Further, if one assumes that all these base stations are accessible to a central control unit via a back haul link, depending on the load, some of these devices can be deactivated to conserve their battery life.

In our current work, we derive optimal policies to conserve energy in two scenarios. 1) A central control unit, which, depending on the load in the system can deactivate (eg. switched OFF) certain base stations to reduce overall energy conservation in the system, but, with an objective to maximize the expected throughput. 2) Each base station can derive its policy independent of its neighbors to minimize an average cost metric (for example, its buffer occupancy), while keeping a lower bound on the fraction of time it is deactivated (eg. idle). To address these problems, we use tools from Multimodularity.

The natural counterpart of convex functions over integer sets turn out to be the so called Multimodular functions ([3]) and for such functions we have indeed the property that local minima are global minima. The property of Multimodularity can handle the control of discrete events and we utilize the same to obtain optimal activation policies for base stations. These tools were earlier used in the context of stochastic control of Queuing systems ([4] and references therein). In a recent work [5], while addressing the problem of energy limited wireless handsets, the authors use tools from Multimodularity to address open loop control and establish optimality of bracket sequences based control.

We consider a regular network, wherein the base stations form a linear network and here the tools of Multimodularity fit in. Tools like stochastic geometry can be used to address networks which have base stations and mobiles distributed stochastically according to some given process (for eg. Poisson) and this study is not addressed here. Our objective is to obtain open loop policies. The main results of our paper are

1) We show that the cost structure for the both centralized and decentralized scenarios is Multimodular.

2) For the central control, among all feasible policies with at least an asymptotic fraction $\rho$ of the base stations being deactivated, the bracket policy with rate $\rho$ is optimal.

3) For the decentralized control, among all feasible policies with the BS deactivated for at least an asymptotic fraction $\rho$ of the time, the bracket policy with rate $\rho$ is optimal.

4) In both the cases, the optimal policy is given by ( $\lfloor x\rfloor$ denotes the largest integer smaller or equal to $x$ )

$$
a_{n}=\lfloor n \rho\rfloor-\lfloor(n-1) \rho\rfloor .
$$

Here, $a_{n}=1(0)$, if the $n$-th BS in the centralized case or BS in the $n$-th time slot in the decentralized case is deactivated (activated).

5) The optimal policies depend only upon the conservation factor $\rho$ and are independent of all other system parameters, for example path loss coefficient, power per transmission, etc.

In both the problems, we call $\rho$, the switch OFF fraction ${ }^{1}$ (the total fraction of base stations to be deactivated in central

\footnotetext{
${ }^{1}$ In this article, we shall use switch OFF and ON to mean deactivation and activation. In our context, deactivation in central control means that the BS is switched OFF and for the decentralized control, this means that the BS is in Idle mode.
} 
control or the fraction of time a base station is deactivated in decentralized control), as the conservation factor. In the first case, this is decided by the central control based on system statistics (like traffic type, load distribution, QoS, time of hour, day of week, power saving, etc.), while in the decentralized case, it is derived based on resources available and the QoS setting (memory, power saving, waiting time, sojourn time, etc.). Further, in our analysis, we assume that this factor is known a priori.

Organization of the paper: In Section II, we introduce Multimodularity concepts and the tools relevant to this paper. In Section III, we address the central control problem, while Section IV studies the decentralized control. We conclude our work in Section V.

Notations: Bold letters, for example a, represent an infinite sequence while $\mathbf{a}_{j}^{k}$ represents a part of this sequence defined by $\left(a_{j}, \cdots, a_{k}\right)$. Let $\mathbb{1}_{1}^{n}$ represent a $n$ vector of all ones while 11 represents the infinite sequence of all ones. In the sections, where the infinite sequences are not used and where all the vectors used are of the same length, then bold letters (for example a) themselves are used to represent the finite length vectors. $\underline{\mathrm{im}}, \varlimsup$ im is limit infimum and supremum, respectively.

\section{Multimodularity}

Multimodularity can be used to address a wide class of control problems over sequences of integer numbers. Multimodular functions turn out to be the natural counter part of Convex functions, in the case of integer valued functions, for which the existence of a local minima guarantees a global minima. Also, they induce a particular form of optimal policies, which turn out to be very regular, and are described by the well known bracket sequences. We reproduce the related definitions (see [4] for general definitions) specific to the spaces considered in this paper.

In this section, we use notation a to represent the $N$-length vector $\mathbf{a}_{1}^{N}$ as here we do not need the infinite length sequences. if

Definition 1: A function $f:\{0,1\}^{N} \rightarrow R$ is Multimodular

$$
f(\mathbf{a}+\mathbf{v})+f(\mathbf{a}+\mathbf{u}) \geq f(\mathbf{a})+f(\mathbf{a}+\mathbf{u}+\mathbf{v})
$$

for all $\mathbf{a} \in\{0,1\}^{N}$ and for all $\mathbf{u}, \mathbf{v} \in \mathcal{F}$ with $\mathbf{u} \neq \mathbf{v}$ and such that $\mathbf{a}+\mathbf{u}, \mathbf{a}+\mathbf{v}, \mathbf{a}+\mathbf{u}+\mathbf{v} \in\{0,1\}^{N}$.

The Multimodular base $\mathcal{F}$ contains the vectors $\left\{-\mathbf{e}_{1}, \mathbf{s}_{2}, \mathbf{s}_{3}, \cdots, \mathbf{s}_{N}, \mathbf{e}_{N}\right\}$, where,

$$
\begin{aligned}
& -\mathbf{e}_{1}=\left(\begin{array}{llllllll}
-1 & 0 & 0 & 0 & 0 & \ldots & 0 & 0
\end{array}\right), \\
& \mathbf{s}_{2}=\left(\begin{array}{lllllllll}
1 & -1 & 0 & 0 & 0 & \ldots & 0 & 0
\end{array}\right), \\
& \mathbf{s}_{3}=\left(\begin{array}{lllllllll}
0 & 1 & -1 & 0 & 0 & \ldots & 0 & 0
\end{array}\right), \\
& \cdots \\
& \mathbf{s}_{N}=\left(\begin{array}{lllllllll}
0 & 0 & 0 & 0 & 0 & \ldots & 1 & -1
\end{array}\right) \text { and } \\
& \mathbf{e}_{N}=\left(\begin{array}{llllllll}
0 & 0 & 0 & 0 & 0 & \ldots & 0 & 1
\end{array}\right)
\end{aligned}
$$

Definition 2: The bracket sequence $\mathbf{a}(\rho, \theta):=\left\{a_{n}(\rho, \theta)\right\}$ with rate $\rho \in[0,1)$ and initial phase $\theta \in[0,1)$ is defined as

$$
a_{n}(\rho, \theta)=\lfloor n \rho+\theta\rfloor-\lfloor(n-1) \rho+\theta\rfloor
$$

In this paper we mainly use Theorem 6, pp. 25, [4] which establishes the optimality of bracket sequences and the same is reproduced here.

Theorem 1: A bracket sequence $\mathbf{a}(\rho, \theta)$ for any $\theta \in[0,1)$ minimizes the cost

$$
\varlimsup_{N \rightarrow \infty} \frac{1}{N} \sum_{n=1}^{N} f_{n}\left(a_{1}, \cdots, a_{n}\right)
$$

over all the sequences that satisfy

$$
\varliminf_{N \rightarrow \infty} \frac{1}{N} \sum_{n=1}^{N} a_{n} \geq \rho,
$$

when $\rho \in[0,1)$, under the following assumptions:

A. $1 f_{n}$ is Multimodular $\forall n$.

A. $2 f_{n}\left(a_{1}, \ldots, a_{n}\right) \geq f_{n-1}\left(a_{2}, \ldots, a_{n}\right), \forall n>1$ and

A.3 for any sequence $\left\{a_{n}\right\}, \exists$ a sequence $\left\{b_{n}\right\}$ such that $\forall n, m$ with $n>m$,

$$
f_{n}\left(b_{1}, \ldots, b_{n-m}, a_{1}, \ldots, a_{m}\right)=f_{m}\left(a_{1}, \ldots, a_{m}\right)
$$

A. 4 for every $n$, the functions $f_{n}\left(a_{1}, \cdots, a_{n}\right)$ are increasing in $a_{i}$ for every $i$.

\section{Centralized optimal CONTROL}

We have uniformly placed points on a line, which are separated by a distance $d$. Each point can potentially contain a $\mathrm{BS}$ and or a mobile. The mobile at any point is active (i.e., has a communication request) with probability $q$ and this process is identical and independent across the space. Some of the base stations are switched OFF to optimize the battery performance. Every BS that is $\mathrm{ON}$ is associated to some of the mobiles based on the nearest distance criterion. Further the BS uses directional antennae and can only communicate with the users to its right. The throughput at the base station from a mobile located at distance $r$ is given by

$$
\theta=q \log \left(1+\frac{p r^{-\beta}}{\sigma^{2}}\right),
$$

where $p, r, \beta, \sigma^{2}$ respectively represent the transmit power from mobile, distance between BS and mobile, path loss factor and the noise variance. Further we assume that there is no intra or inter-cell interference. Note that the throughput is achieved only when the mobile has a request, which occurs with probability $q$.

Our goal is to find an optimal switch OFF pattern of the base stations so as to maximize the sum of the expected throughputs of all the mobiles when one has to at least switch OFF a fraction $\rho$ of the base stations. The conservation factor $\rho$ at any time period will be decided by the network based on the load and for a given $\rho$ the network prefers to adopt an optimal ON-OFF pattern.

We obtain this answer in the asymptotic limit as $N$, the number of points in the space tend to $\infty$. Consider a sequence $\mathbf{a} \in\{0,1\}^{\infty}$ to represent the control sequence in the following sense: $a_{i}$, the action at $i^{\text {th }}$ point is 1 if $\mathrm{BS}$ is switched OFF and 0 if BS is ON. The goal is to find an optimal sequence $\mathbf{a}^{*}$ which maximizes the expected throughput (defined via Cesaro 


\begin{tabular}{|l|c|c|c|c|c|c|}
\hline Sequence a & 100100100 & 101000100 & 010010010 & 101000010 & 100010001 & 001001001 \\
\hline Throughput $\Theta$ & 5.1 & 5.0 & 4.9 & 4.86 & 4.6 & 4.4 \\
\hline
\end{tabular}

Table 1: Expected system throughput $\Theta=\frac{1}{N} \sum_{1}^{N} \theta_{N}(\mathbf{a})$ for different sequences.

The underlined sequence is the bracket sequence which optimizes the throughput.

limit) such that the total fraction of the base stations, that are switched OFF, is lower bounded by $\rho$. We assume that the system starts at point 0 where a BS is always switched $\mathrm{ON}$ and we control the ON-OFF status of the remaining base stations, i.e., the ones starting from point 1 onwards. We neglect the throughput due to the mobile at 0 as it does not contribute to optimization. Thus, we maximize

$$
\begin{array}{r}
\max _{\mathbf{a}} \varlimsup_{N \rightarrow \infty} \frac{1}{N} \sum_{n=1}^{N} \theta_{n}(\mathbf{a}) \\
\text { subject to } \underset{N \rightarrow \infty}{\underline{\lim }} \frac{1}{N} \sum_{n=1}^{N} a_{n} \geq \rho
\end{array}
$$

where $\theta_{n}(\mathbf{a})$ is the throughput due to the mobile at position $n$ (which depends upon the position of the nearest base station). This depends upon the sequence a and equals (note the base station at point 0 is always $\mathrm{ON}$ ):

$$
\theta_{n}(\mathbf{a})=q \log \left(1+\frac{p\left(1+r_{n}^{2}\right)^{-\beta / 2}}{\sigma^{2}}\right)
$$

where,

$$
r_{n}:= \begin{cases}n d & \text { if } \mathbf{a}_{1}^{n}=\mathbb{1}_{1}^{n} \\ \inf _{1 \leq j \leq n}\left\{|n-j| d: a_{j}=0\right\} & \text { else. }\end{cases}
$$

We use tools from Multimodularity [4] to address this problem. The related definitions are summarized in section II. We use Theorem 1 and obtain (proof is in Appendix A)

Theorem 2: The function $f_{n}\left(\mathbf{a}_{1}^{n}\right):=-\theta_{n}(\mathbf{a})$ is Multimodular for every $n$. Further, the centralized problem (3) is optimized by a bracket sequence (2),

$$
\mathbf{a}^{*}=\mathbf{a}(\rho, \theta) \text { for some } \theta \in[0,1) . \diamond
$$

From the above theorem it is clear that the optimal sequence depends only upon the conservation factor $\rho$ and nothing else. We now give some examples of bracket sequences.

Example 1: The bracket sequence 100100100 maximizes the expected throughput for $(\rho, \theta)=(0.33,0.9)$.

Example 2: The bracket sequence 1001001000 maximizes the expected throughput for $(\rho, \theta)=(0.3,0.9)$.

If the factor $\rho$ is rational, then the sequence is periodic ([3]). In this case the optimal policy is to switch OFF the base stations in a periodic fashion, for example with $\rho=0.33$ one needs to switch OFF every third BS. The optimality of bracket sequence is established in the limit $N$, the number of points, tending to infinity. This would imply the bracket sequence would be nearly optimal for systems with large $N$. It would also be optimal for not so large values of $N$ and this is established using a numerical example. We consider a system with $N=9$ points in the space, $\rho=1 / 3$ and obtain the optimal control sequence by exhaustive search. The results are tabulated in Table 1 . We observe that the system throughput is maximized again for the bracket sequence 100100100 .

\section{DECENTRALIZED OPTIMAL CONTROL}

We have base stations deployed in a network. Each BS is powered by a battery and can transmit up to a maximum of $B$ bytes during a transmission opportunity, which are slotted over time. The BS can either be in an active (ON) state where it transmits packets or in an idle state where it shuts OFF its activity to conserve the battery. Note that whenever a BS enters idle (OFF) mode, more number of packets get stored in the buffer and the buffer occupancy cost increases. What is an optimal policy to minimize the average buffer occupancy such that the BS is switched OFF at least for a fraction $\rho$ of the time?

Let $\mathbf{a}=\left\{a_{t}\right\}_{t \geq 1}, a_{t} \in\{0,1\}$, be a sequence of controls such that $a_{t}=1$ indicates BS is OFF at the $t^{t h}$ time slot and $a_{t}=0$ indicates that the BS can serve at maximum $B$ packets. With any general ON-OFF policy, a, the buffer occupancy, $x_{t}$ evolves as

$$
x_{t}(\mathbf{a})=\left(x_{t-1}(\mathbf{a})-\left(1-a_{t}\right) B\right)^{+}+w_{t}
$$

and it begins with $x_{0}=w_{0}$.

In the above, $w_{t}$ represents the new arrivals in the $t$-th time slot. We assume $\left\{w_{t}\right\}_{n \geq 0}$ is an Identically and Independently distributed (IID) sequence and that it is bounded by $B$, i.e., $w_{t} \leq B$ with probability one. We now have the following problem of minimizing

$$
\begin{array}{r}
\min _{\mathbf{a}} \varlimsup_{T \rightarrow \infty} \frac{1}{T} \sum_{t=1}^{T} E\left[x_{t}(\mathbf{a})\right] \\
\text { subject to } \quad \underline{\lim _{T \rightarrow \infty}} \frac{1}{T} \sum_{t=1}^{T} a_{t} \geq \rho .
\end{array}
$$

One needs to choose the conservation factor, $\rho$ such that

$$
B(1-\rho)>E[W] .
$$

This has to be done to ensure that the system can be stable at least for some of the control sequences a. With the above condition, the system for example is stable for all those sequences whose switch OFF fraction exactly equals $\rho$. We again use the Multimodularity Theorem 1 and obtain (proof is in Appendix B)

Theorem 3: The function $f_{t}\left(\mathbf{a}_{1}^{t}\right):=E\left[x_{t}(\mathbf{a})\right]=E\left[x_{t}\left(\mathbf{a}_{1}^{t}\right)\right]$ is Multimodular for every $t$. And, the decentralized problem (8) is optimized by a bracket sequence.

$$
\mathbf{a}^{*}=\mathbf{a}(\rho, \theta) \text { for some } \theta \in[0,1) . \diamond
$$

Thus the optimal sequence is again a bracket sequence which depends only on $\rho$, the conversation factor. 
Hence, in both centralized and decentralized problems, we have similar optimal control pattern which depends only upon the conservation factor $\rho$ and none of the other design parameters.

\section{CONCLUSIONS}

In this paper, we derived energy conserving policies for Base stations in regular green networks using tools from Multimodularity. We considered two example scenarios and show how the cost functions are Multimodular. In the first case, for the case of central control, we derived the optimal open loop policies so as to maximize the expected throughput of the system given that at least a certain percentage of Base stations are deactivated (switched OFF). In the second case, we derived optimal open loop polices, which each base station can employ in a decentralized manner to minimize buffer occupancy costs, while keeping the long term average fraction of the BS deactivation (idle mode) time at least above a given threshold. We established the optimality of bracket policies for both the cases. We established that these regular sequences optimize the (respective) performance(s) and these sequences depend only upon the conservation factor and nothing else. We conclude that Multimodularity can be applied in several interesting example scenarios to derive optimal control in Green Networks.

\section{REFERENCES}

[1] Han, C., Harrold, T., Krikidis, I., Ku, I., Le, T. A., Videv, S., Zhang, J., Armour, S., Grant, P. M., Haas, H., Hanzo, L., Nakhai, M. R., Thompson, J. S. and Wang, C. X. "Green Radio: Radio Techniques to Enable Energy Efficient Wireless Networks", IEEE Communications Magazine Special Issue: Green Communications", (In Press)

[2] J. Hoydis and M. Debbah, "Green, Cost-effective, Flexible, Small Cell Networks", IEEE ComSoc MMTC E-Letter Special Issue on Multimedia Over Femto Cells, vol. 5, no. 5, pp. 23-26, Sep. 2010

[3] B. Hajek, "External splittings of point processes", Mathematics of operation research, 10(4):543-546, 1985

[4] E. Altman, B. Goujal, A. Hordijk, "Discrete-Event Control of Stochastic Networks: Multimodularity and Regularity", Lecture Notes in Mathematics, No. 1829, 2001, Springer.

[5] V. S. Borkar, A. A. Kherani, B. J. Prabhu, "Closed and Open Loop Optimal Control of Buffer and Energy of a Wireless Device", Proceedings of WiOpt 2005, Apr 3-7, Trentino, Italy.

[6] V. S. Borkar, A. A. Kherani, B. J. Prabhu, "Control of Buffer and Energy of a Wireless Device: Closed and Open Loop Approaches", INRIA Research Report RR-5414, Dec 2004.

[7] Stephane Gaubert et. al., "Methods and Applications of $(\max ,+)$ Linear Algebra", INRIA research report RR-3088, Jan 1997. Available at http://hal.inria.fr/inria-00073603/en/

[8] R. R. Weber and S. Stidham, "Optimal control of service rates in a network of queues", Advances in applied probability, 19:202-218, 1987.

[9] P. Glasserman and D. D. Yao, "Monotone structures in discrete event systems", Wiley, New York, 1994.

\section{APPENDiX A: Proofs RELATED TO CENTRALIZED CONTROL}

Proof of Theorem 2: The proof is obtained using Theorem 1 of section II. By Theorem 4 given below, the function

$$
f_{n}\left(a_{1}, \cdots, a_{n}\right)=-\theta_{n}(\mathbf{a})
$$

is Multimodular and hence assumption A.1 of Theorem 1 is satisfied.
Given $\mathbf{a}_{1}^{n}$, define $a_{0}=0$ and then define

$$
n_{b}:=\arg \inf _{0 \leq j \leq n}\left\{|n-j|: a_{j}=0,\right\}
$$

to denote the index of the nearest base station in the left for the mobile at point $n$. When $n_{b}>1$, assumption $\mathbf{A} .2$ is satisfied as then

$$
f_{n}\left(\mathbf{a}_{1}^{n}\right)=f_{n-1}\left(\mathbf{a}_{2}^{n}\right)=f\left(\mathbf{a}_{n_{b}}^{n}\right) .
$$

When $n_{b}=1$ (i.e., when $\mathbf{a}_{1}^{n}=(0,1, \cdots, 1)$ ) the equality still holds in A.2 by the definitions (5)-(6). When $\mathbf{a}_{1}^{n}=1_{1}^{n}$, then the assumption A.2 is satisfied with inequality as

$$
\theta_{n}\left(\mathbb{1}_{1}^{n}\right)<\theta_{n-1}\left(\mathbb{1}_{1}^{n-1}\right) .
$$

Assumption A. 3 is satisfied by taking $\left\{b_{n}\right\}$ to be all zeros. For any $n$ and for any $1 \leq i \leq n$, clearly with $a_{j}$ for all $j \neq i$ fixed

$$
\begin{aligned}
f_{n}\left(a_{1}, a_{2}, \cdots, a_{i-1},\right. & \left.1, a_{i+1}, \cdots, a_{n}\right) \\
& \geq f_{n}\left(a_{1}, a_{2}, \cdots, a_{i-1}, 0, a_{i+1}, \cdots, a_{n}\right)
\end{aligned}
$$

and hence the assumption A.4 is satisfied. Thus all the hypothesis of Theorem 1 are satisfied and hence the theorm follows by Theorem 1 .

Theorem 4: For every $n, f_{n}$ is Multimodular.

Proof: All the sequences in this proof are $n$ length vectors and hence we use the short notation $\mathbf{a}$ in place of $\mathbf{a}_{1}^{n}$. Consider any sequence $\mathbf{a}$. We need to show for $\mathbf{u} \neq \mathbf{v} \in \mathcal{F}$ (see section II for definitions) $f_{n}$ satisfies

$$
\begin{gathered}
f_{n}(\mathbf{a}+\mathbf{u})+f_{n}(\mathbf{a}+\mathbf{v}) \geq f_{n}(\mathbf{a})+f_{n}(\mathbf{a}+\mathbf{u}+\mathbf{v}) \\
\text { whenever, } \mathbf{a}+\mathbf{u}, \mathbf{a}+\mathbf{v}, \mathbf{a}+\mathbf{u}+\mathbf{v} \text { are all in }\{0,1\}^{n} .
\end{gathered}
$$

Without loss of generality let $\mathbf{v}=\mathbf{s}_{j}$ (see section II for definition of $\mathbf{s}_{j}$ ), which when added to a results in $\mathbf{a}+\mathbf{v}$ in which the ON position of the base station is shifted from $j-1$ location to location $j$. Since we can only consider such $\mathbf{s}_{j}$ for which $\mathbf{a}+\mathbf{s}_{j}$ is in $\{0,1\}^{n}$ the sequence a should have

$$
a_{j-1}=0 \text { and } a_{j}=1 .
$$

Further,

$$
\begin{gathered}
(a+v)_{j-1}=1, \quad(a+v)_{j}=0 \\
\text { and } a_{i}=(a+v)_{i} \text { for every } i \neq j \text { or } i \neq j-1 .
\end{gathered}
$$

Thus, addition of vector $\mathbf{v}$ to a results in changes in the base station associations and hence the throughputs, only for the the mobiles located in $\left(j-1, \cdots, \eta_{j}^{r}-1\right)$, where $\eta_{j}^{r}$ is defined as the nearest base station to the right of the location $j$ that is switched $\mathrm{ON}$ in a:

$\eta_{j}^{r}:= \begin{cases}\arg \inf _{n>j}\left\{|n-k|: a_{k}=0\right\} & \text { if set is non empty } \\ n+1 & \text { else. }\end{cases}$

Hence,

$$
\begin{aligned}
f_{n}(\mathbf{a})-f_{n}(\mathbf{a}+\mathbf{v}) & =\sum_{k=1}^{n} \theta_{k}(\mathbf{a})-\sum_{k=1}^{n} \theta_{k}(\mathbf{a}+\mathbf{v}) \\
& =\sum_{k=j-1}^{\eta_{j}^{r}-1} \theta_{k}(\mathbf{a})-\theta_{k}(\mathbf{a}+\mathbf{v}) .
\end{aligned}
$$


Let $\mathbf{u}=\mathbf{s}_{l}$ with $l>j+1(l \neq j+1$ as then it is not possible that both $\mathbf{a}+\mathbf{u}$ and $\mathbf{a}+\mathbf{v}$ are in $\{0,1\}^{n}$ ). The addition of $\mathbf{u}$ to $\mathbf{a}$ will introduce changes in mobile throughputs only at locations $\left(l-1, \cdots, \eta_{l}^{r}\right)$, which do not overlap with locations changed by $\mathbf{v},\left(j-1, \cdots, \eta_{j}^{r}\right)$. Further, the addition of $\mathbf{v}$ to $\mathbf{a}+\mathbf{u}$ also changes the mobile throughputs only in locations $(j-$ $1, \cdots, \eta_{j}^{r}$ ) (w.r.t. the mobile throughputs under $\mathbf{a}+\mathbf{u}$ ). Because of the independence of the locations of the changes due to $\mathbf{v}$ and $\mathbf{u}$ and because the mobile throughputs only depend upon the distance w.r.t. the serving BS, the mobile throughput changes from $\mathbf{a}+\mathbf{u}$ to $\mathbf{a}+\mathbf{u}+\mathbf{v}$ will be same as that when $\mathbf{a}$ is changed to $\mathbf{a}+\mathbf{v}$. Thus,

$$
\begin{aligned}
f_{n}(\mathbf{a} & +\mathbf{u})-f_{n}(\mathbf{a}+\mathbf{u}+v) \\
& =\sum_{k=j-1}^{\eta_{j}^{r}} \theta_{k}(\mathbf{a}+\mathbf{u})-\theta_{k}(\mathbf{a}+\mathbf{u}+\mathbf{v}) \\
& =\sum_{k=j-1}^{\eta_{j}^{r}} \theta_{k}(\mathbf{a})-\theta_{k}(\mathbf{a}+\mathbf{v})=f_{n}(\mathbf{a})-f_{n}(\mathbf{a}+\mathbf{v}) .
\end{aligned}
$$

The second vector $\mathbf{u}$ can either be $\mathbf{s}_{l}$ with $l>j+1$ or $l<j-1$ (as both $\mathbf{a}+\mathbf{u}$ and $\mathbf{a}+\mathbf{v}$ have to be in $\{0,1\}^{n}$ ) or $\mathbf{u}$ can be $\mathbf{e}_{n}$ when $j<n$ or it can $\mathbf{e}_{1}$ when $j>1$. In all the combinations, addition of vectors $\mathbf{u}$ and $\mathbf{v}$ results in changes to the base station association at independent locations as above. Thus for any $\mathbf{u} \neq \mathbf{v}$, as in (10), one can show that

$$
f_{n}(\mathbf{a}+\mathbf{u})+f_{n}(\mathbf{a}+\mathbf{v})=f_{n}(\mathbf{a})+f_{n}(\mathbf{a}+\mathbf{u}+\mathbf{v}) .
$$

\section{APPENDIX B: PROOFS RELATED TO DECENTRALIZED CONTROL}

Proof of Theorem 3: The proof is again obtained using Theorem 1 of section II. By Theorem 5, the function

$$
g_{t}\left(a_{1}, a_{2}, \cdots, a_{t}\right)=x_{t}(\mathbf{a})
$$

is Multimodular for every sample path of the arrival sequence, $\mathbf{w}_{0}^{t}=\left(w_{0}, w_{1}, \cdots, w_{t}\right)$. The sample path wise Multimodularity implies the Multimodularity of the average function

$$
f_{t}\left(\mathbf{a}_{1}^{t}\right)=E\left[x_{t}\left(\mathbf{a}_{1}^{t}\right)\right]
$$

and thus the first part of the theorem is established as well as the assumption A.1 is satisfied.

The initial buffer size is $w_{0}$, i.e., $x_{0}(\mathbf{a})=w_{0}$ for all sequences a and all samples. The function value $f_{t}\left(\mathbf{a}_{1}^{t}\right)$ is the average buffer size at $t-1$ time slot, obtained by progressing (Lindley's recursion) $t-1$ time slots using the control sequence $\mathbf{a}_{2}^{t}$ and when initial buffer size is given by $x_{1}\left(a_{1}\right) \geq w_{1}$ while the function value $f_{t-1}\left(\mathbf{a}_{2}^{t}\right)$ is the average buffer size at the same time slot obtained again by progressing $t-1$ time slots using the same control sequence $\mathbf{a}_{2}^{k}$ but now with initial buffer size equal to $w_{0}$. Note that $w_{1}$ is distributed same as $w_{0}$ and hence

$$
f_{t}\left(\mathbf{a}_{1}^{t}\right) \geq f_{t-1}\left(\mathbf{a}_{2}^{t}\right) .
$$

Thus, the assumption A.2 is satisfied.

For assumption A.3 take $\left\{b_{t}\right\}$ to be an all zero sequence. When the control sequence is all zeros, i.e., the BS serves in all the time slots, since the maximum number of arrivals in a slot is $B$, the buffer size at the end of every slot $t$, will exactly be $w_{t}$, the new arrivals. Thus the function value $f_{t}\left(\mathbf{b}_{1}^{t-\tau}, \mathbf{a}_{1}^{\tau}\right)$ represents the average buffer size after $\tau$ time slots when the control sequence is $\mathbf{a}_{1}^{\tau}$ and when the initial buffer size is $w_{t-\tau}$ while $f_{\tau}\left(\mathbf{a}_{1}^{\tau}\right)$ represents the same after $\tau$ time slots and with the same control sequence $\mathbf{a}_{1}^{\tau}$ but with initial buffer size $w_{0}$ and hence the two average values are equal. Thus, assumption A. 3 is satisfied.

Clearly, assumption A.4 is also satisfied and hence the theorem follows by Theorem 1 .

Theorem 5: For every $t$ and for every sample path $\mathbf{w}_{0}^{t}$, the function $g_{t}$ is Multimodular.

Proof: In [5], while addressing the problem of energy limited wireless handsets, the authors show that the function $x_{t}\left(\mathbf{a}_{1}^{t}\right)$ is Multimodular for every sample path of the arrival sequence $\left\{w_{t}\right\}$. The functions used in describing their Cesaro limit (see [5]) are exactly the same as the functions $\left\{x_{t}\right\}$ of the decentralized problem. The sample path wise Multimodularity is proved as Theorem 17, page 6 [5] (details of this proof are in their technical report, Theorem 20, [6]). The proof there is little difficult to read and hence we provide a brief overview of the same below:

If $x_{t-1} \leq B$ then $a_{t}=0$ results in an empty queue and $x_{t}=w_{t}$. On the other hand, if $x_{t-1}>B$, some part $\Delta x_{t-1}=$ $x_{t-1}-B$, remains in the queue and thus

$$
x_{t}=\Delta x_{t-1}+w_{t} .
$$

Using this one can show that

$$
\begin{array}{r}
x_{t}(\mathbf{a})=x_{t}\left(\mathbf{a}+\mathbf{s}_{j}\right) \text { if } x_{j-2}>B \\
\text { and } x_{t}(\mathbf{a}) \geq x_{t}\left(\mathbf{a}+\mathbf{s}_{j}\right) \text { if } x_{j-2} \leq B
\end{array}
$$

Without loss of generality, let $\mathbf{v}=\mathbf{s}_{j}$ with $j=2, \cdots, t$. Then by defintion 1 of section II, there are three possible cases: i) $\mathbf{u}=-\mathbf{e}_{1}$, ii) $\mathbf{u}=\mathbf{s}_{k}, k>j$ and iii) $\mathbf{u}=\mathbf{e}_{t}$ and we need to show for every combination that the equation (1) is satisfied, by the function $g_{t}=x_{t}$, to complete the Multimodularity proof. In the following we present the proof for the case (ii) and the remaining cases are much simpler and follow similar logic.

Let $\mathbf{u}=\mathbf{s}_{k}$ with $k>j$. Note that

$$
x_{l}(\mathbf{a}+\mathbf{u})=x_{l}(\mathbf{a}) \text { for all } l<k-1 .
$$

Thus, $x_{j-2}(\mathbf{a}) \geq B$ if and only if $x_{j-2}(a+v) \geq B$. Note further that this case is possible only if

$$
a_{j-1}=0, a_{j}=1 \text { and } a_{k-1}=0, a_{k}=1 .
$$

Case a: If $x_{j-2}(\mathbf{a}) \geq B$ then $x_{j-2}(a+v) \geq B$ and so

$$
x_{t}(\mathbf{a}+\mathbf{v})=x_{t}(\mathbf{a}) \text { and } x_{t}(\mathbf{a}+\mathbf{u}+\mathbf{v})=x_{t}(\mathbf{a}+\mathbf{u}) .
$$

Thus (1) is satisfied with equality.

Case b: If $x_{j-2}(\mathbf{a})<B$.

(i) In additon if $x_{k-2}(\mathbf{a}) \geq B$ and $x_{k-2}(\mathbf{a}+\mathbf{v}) \geq B$ then again

$$
x_{t}(\mathbf{a}+\mathbf{u})=x_{t}(\mathbf{a}) \text { and } x_{t}(\mathbf{a}+\mathbf{v}+\mathbf{u})=x_{t}(\mathbf{a}+\mathbf{v})
$$

and so again (1) is satisfied with equality. 
(ii) In addition if $x_{k-2}(\mathbf{a}) \geq B$ and $x_{k-2}(\mathbf{a}+\mathbf{v})<B$ then we have

$$
x_{t}(\mathbf{a}+\mathbf{u})=x_{t}(\mathbf{a}) \text { and } x_{t}(\mathbf{a}+\mathbf{v}+\mathbf{u}) \leq x_{t}(\mathbf{a}+\mathbf{v})
$$

and now (1) is satisfied, but need not be with equality.

(iii) Now if $x_{k-2}(\mathbf{a})<B$ and $x_{k-2}(\mathbf{a}+\mathbf{v})<B$ then because $a_{k-1}=0$ so is $(a+v)_{k-1}=0$ (the controls in $a$, $a+v$ are same after $j+1), x_{k-1}(\mathbf{a})=x_{k-1}(\mathbf{a}+\mathbf{v})$ as they both result only because of new arrivals at $k-1$ and older ones (which might be different) were flushed out completely. Since there is no difference in both the controls $\mathbf{a}, \mathbf{a}+\mathbf{v}$ after the time point $j$,

$$
x_{t}(\mathbf{a})=x_{t}(\mathbf{a}+\mathbf{v}) .
$$

From (11) we do have

$$
x_{t}(\mathbf{a}+\mathbf{u}) \geq x_{t}(\mathbf{a}+\mathbf{u}+\mathbf{v})
$$

and so again (1) is satisfied. 